\title{
Krüppel-like factor 4: A new potential biomarker of lung cancer
}

\author{
MARIE CLAUDE FADOUS-KHALIFÉ ${ }^{1,2}$, NIJEZ ALOULOU ${ }^{1}$, MAJIDA JALBOUT ${ }^{1,3}$, \\ JOSEPH HADCHITY ${ }^{4}$, GEORGES AFTIMOS ${ }^{5}$, FRANÇOIS PARIS ${ }^{6}$ and ELIE HADCHITY ${ }^{1}$ \\ ${ }^{1}$ Anti-Tumor Therapeutic Targeting Laboratory, Faculty of Sciences, Lebanese University, Hadath; \\ ${ }^{2}$ Notre Dame De Secours University Hospital, Byblos; ${ }^{3}$ Immunogenetic Pathology Laboratory, Faculty of Sciences, \\ Lebanese University, Fanar; ${ }^{4}$ Department of Surgery, Sainte Thérèse Hospital, Hadath; ${ }^{5}$ National Institute of Pathology, \\ Baabda, Lebanon; ${ }^{6}$ Endothelium Radiobiology and Targeting, UMR Inserm 892, Cancer Research Center, Nantes, France
}

Received December 10, 2015; Accepted April 7, 2016

DOI: $10.3892 / \mathrm{mco} .2016 .883$

\begin{abstract}
Lung cancer is most prevalent human cancer worldwide. However, no molecular markers are currently available for predicting lung cancer prognosis. Therefore, identifying novel biomarkers may be useful for improving clinical diagnosis and patient stratification. Krüppel-like factor 4 (KLF4) is a transcription factor with opposing roles in different human cancers. Its overexpression in several cancers is correlated with a poor prognosis. However, the expression and role of KLF4 in lung cancer remains to be elucidated. The aim of this study was to determine the profile of KLF4 expression in different types of lung cancer. The KLF4 protein expression level was tested and evaluated by immunohistochemical analysis in 47 lung tumors and normal tissues, and then correlated with clinical characteristics. A differential expression of KLF4 was observed between normal tissue and each of the lung cancer types. A significant decrease in KLF4 expression was observed in non-small-cell lung cancer (NSCLC) compared with that in normal tissue, while significant overexpression was detected in small-cell lung cancer. Furthermore, a higher rate of expression was observed in stage II, III and IV disease compared with stage I disease in NSCLC tissues. KLF4 expression was not found to be associated with age or gender. Our results suggested that the KLF4 protein level may be a potential biomarker in patients with advanced lung cancer.
\end{abstract}

\section{Introduction}

Lung cancer is the most common malignancy affecting both genders and remains the main cause of cancer-related mortality worldwide (1). Despite advances in diagnosis and treatment of lung cancer, it remains a disease with high morbidity and

Correspondence to: Professor Elie Hadchity, Anti-Tumor Therapeutic Targeting Laboratory, Faculty of Sciences, Lebanese University, Hadath Educational Campus, Hadath, Lebanon

E-mail: eliehadchity@hotmail.com

Key words: Krüppel-like factor 4, immunohistochemistry, prognostic marker, lung cancer mortality. Lung cancers are classified according to histological type and this classification has important implications for the clinical management and prognosis of this disease. The two main histological groups are non-small-cell lung cancer (NSCLC) and small-cell lung cancer (SCLC). Approximately $85 \%$ of lung tumors are NSCLCs. NSCLC includes three major histological subtypes: Adenocarcinoma, squamous cell carcinoma and large-cell carcinoma (2). Despite the new therapeutic approaches, the overall survival of patients with lung cancer remains low. The 5-year survival for SCLC is lower compared with that of NSCLC (6 vs. 18\%, respectively). Therefore, the identification of highly sensitive and specific biomarkers that highlight pathological changes early during the course of the disease, in order to allow timely clinical intervention, is crucial. A better understanding of biomarkers associated with lung cancer may be of clinical value in improving treatment selection and prognostication, and may even set the base for the development of future novel targeted therapies.

Krüppel-like factors (KLFs) are a family of evolutionarily conserved mammalian zinc finger transcription factors, named after their homology with Krüppel, which is a Drosophila melanogaster protein (3). KLFs are involved in a number of important cellular processes, such as growth, development, differentiation, proliferation and apoptosis (4-6). KLF4 (also referred to as gut-enriched KLF or GKLF) is one of the first KLF family members identified $(7,8)$.

KLF4 is a transcription factor expressed in a wide variety of human tissues, which is important for a number of different physiological processes, including development, differentiation and maintenance of normal tissue homeostasis. KLF4 is a bifunctional transcription factor able to either activate or repress transcription using different mechanisms, depending on the target gene. Thus, depending on the cell type or cell context, KLF4 may act either as a tumor suppressor gene or as an oncogene.

KLF4 is implicated as a tumor suppressor gene in the gastrointestinal tract epithelium, as its expression is decreased in human colon and gastric cancers (9-11). The loss of KLF4 is associated with poor survival (10) and it was found to be downregulated in gastric cancer, with evidence of hypermethylation of the 5'-untranslated region and loss of heterozygosity of the KLF4 locus or point mutations in the coding region (12-14). A similar tumor suppressor role is also observed in colorectal 
cancer (9), esophageal cancer (15), lung cancer (16), bladder cancer (17), medulloblastoma (12) and T-cell leukemia (13).

Conversely, KLF4 may function as a transforming oncogene. KLF4-transformed rat kidney epithelial cells exhibit morphological transformation and an increased tumorigenicity in athymic mice (14). Increased KLF4 expression has been reported in human head and neck squamous cell carcinoma and breast cancer $(14,18)$. Moreover, KLF4 expression has been demonstrated to be a poor prognostic factor for early breast cancer and skin cancer $(19,20)$, corroborating its oncogenic role. In the skin, overexpression of KLF4 results in hyperplasia and dysplasia (21), eventually leading to the development of squamous cell carcinoma (22). Whether KLF4 acts as a tumor suppressor or an oncogene is likely determined by differences in cell context, expression patterns of other genes and the chromatin environment of individual cells. However, the mechanism underlying these differences remains unknown.

A recent study demonstrated that KLF4 may function as a tumor suppressor gene in lung cancer. The expression of KLF4 was downregulated in 21 of 25 primary lung cancers and ectopic expression of KLF4 suppressed lung cancer cell proliferation and clonogenic formation in vitro. Moreover, transfection of lung cancer cells with the KLF4 gene also suppressed tumor growth in vivo (16). However, the molecular mechanism underlying the tumor-suppressive function of KLF4 in lung cancer remains to be determined, as only few studies have investigated the role and differences in expression of KLF4 among different histological groups of lung cancer. In this study, the KLF4 protein expression level was investigated in lung tumors (31 adenocarcinomas and 16 SCLCs) and normal tissues and the clinical significance of KLF4 expression for diagnosis and treatment decision-making was evaluated using immunohistochemical analysis.

\section{Materials and methods}

Sample collection and clinicaldata. A total of 47 formalin-fixed paraffin-embedded lung cancer samples (31 adenocarcinomas and 16 SCLCs) and normal tissue samples from healthy donors $(n=13)$ were collected between January, 2014 and July, 2015 from the Department of Pathological Anatomy of the Notre Dame de Secours University Hospital (Byblos, Lebanon) and the National Institute of Pathology (Baabda, Lebanon). All the tumor and normal tissue samples were obtained from surgical specimens of patients with lung cancer. This study was approved by the Institutional Review Board of the Notre Dame de Secours University Hospital.

Immediately following surgical removal, all the tissue samples were fixed in formalin and embedded in paraffin prior to sectioning for histological and immunohistochemical analyses. All the cancer tissue samples were graded by a pathologist and histologically classified. Epidemiological and clinical information were collected from patient records and registries (Table $\mathrm{I}$ ).

Immunohistochemistry and scoring. Paraffin-embedded tissue sections $(4 \mu \mathrm{m})$ were subjected to immunostaining using the Ventana automated stainer (BenchMark XT; Roche Diagnostics GmbH, Mannheim, Germany) at the National
Institute of Pathology (Baabda, Lebanon). The tissue sections were hydrated through xylene and graded ethanols and equilibrated in phosphate-buffered saline prior to undergoing antigen retrieval. Endogenous peroxidase activity was quenched with $0.3 \%$ hydrogen peroxide for $5 \mathrm{~min}$ and the tissue sections were incubated with a mouse monoclonal anti-KLF4 antibody (cat. no. SAB5300069; clone 1E6; Sigma-Aldrich, St. Louis, MO, USA) at a dilution of 1:200 for $1 \mathrm{~h}$ at room temperature. The appropriate secondary antibody was horseradish peroxidase (HRP)-conjugated rabbit anti-mouse IgG (cat. no. A9044; Sigma-Aldrich) at a dilution of 1:200 for $1 \mathrm{~h}$ at room temperature. HRP detection was achieved using 3,3'-diaminobenzidine substrate (Sigma-Aldrich) and the slides were counterstained with hematoxylin.

Immunostaining was blindly evaluated by two investigators (G.A. and E.H.) in an effort to achieve a consensus on staining patterns by light microscopy. A quantitative score was estimated by adding the score of the staining area and that of staining intensity for each case to assess the expression levels of the protein. The quantitative score was estimated by calculating the percentage of immunopositive cells as follows: 0 , no staining of cells in any microscopic fields; $1+,<30 \%$ of cells stained positive; $2+, 30-60 \%$ stained positive; and $3+,>60 \%$ stained positive. The intensity was scored by evaluating the average staining intensity of the positive cells as follows: 0 , no staining; $1+$, mild staining; $2+$, moderate staining; and $3+$, intense staining.

Data analysis. All statistical analyses were performed using SPSS software for Windows, version 18.0 (SPSS Inc., Chicago, IL, USA). A paired t-test was used to compare the KLF4 expression level between tumors and matched normal tissues and among different histological tumor types.

The patients were classified into two groups, namely NSCLC and SCLC. The $\chi^{2}$ test was applied to determine the correlation between the KLF4 level and clinicopathological parameters. $\mathrm{P}<0.05$ was considered to indicate statistically significant differences.

\section{Results}

Patient characteristics. The characteristics of the patients included in this study are summarized in Table I. Lung cancer tissues were obtained from 47 patients, namely 31 cases with NSCLCs (adenocarcinomas) and 16 cases with SCLCs. The median age of the patients was 63 years, and $66.66 \%$ of the patients were male. Epidemiological and clinical information was collected from patient records and registries. All the cancer tissue samples were graded by a pathologist and histologically classified. After diagnosis, $25.8 \%$ of the NSCLC patients were diagnosed as stage I $(n=8), 29.03 \%$ were stage II $(n=9), 22.58 \%$ were stage III $(n=7)$ and $22.58 \%$ were stage IV $(\mathrm{n}=7)$.

KLF4 protein expression in normal and tumor tissues. The profile of KLF4 protein expression in the different types of tissues is presented in Table II and in Fig. 1. In normal tissues, KLF4 was expressed in the nuclei with an overall score of $1.38 \pm 0.6$ (mean \pm standard deviation). In all tumor tissue types, the overall score of KLF4 expression was 1.46 \pm 1.27 . 
Table I. Patient characteristics.

\begin{tabular}{lc}
\hline Characteristics & No. $(\%)$ \\
\hline Total subjects & 60 \\
Healthy & $13(21.66)$ \\
NSCLC & $31(51.66)$ \\
SCLC & $16(26.66)$ \\
Gender & \\
Male & $40(66.66)$ \\
Female & $20(33.33)$ \\
Age in years, median (range) & 63 \\
Healthy & $57(20-87)$ \\
NSCLC & $66(21-84)$ \\
SCLC & $65(23-83)$ \\
Stage (NSCLC) & 8 \\
I & 9 \\
II & 7 \\
III & 7 \\
IV & \\
\hline
\end{tabular}

NSCLC, non-small-cell lung cancer; SCLC, small-cell lung cancer.

Table II. Mean KLF4 protein expression in normal and lung cancer tissues.

\begin{tabular}{lcc}
\hline Tissue type & $\begin{array}{c}\text { Mean KLF4 } \\
\text { protein expression }\end{array}$ & $\begin{array}{c}\text { P-value vs. } \\
\text { normal tissue }\end{array}$ \\
\hline Normal $(\mathrm{n}=13)$ & $1.38 \pm 0.6$ & \\
Cancer $(\mathrm{n}=47)$ & $1.46 \pm 1.27$ & 0.995 \\
NSCLC $(\mathrm{n}=31)$ & $0.7 \pm 0.3$ & 0.02 \\
SCLC $(\mathrm{n}=16)$ & $2.68 \pm 0.46$ & 0.00003 \\
\hline
\end{tabular}

Values are expressed as mean \pm standard deviation. KLF4, Krüppel-like factor 4; NSCLC, non-small-cell lung cancer; SCLC, small-cell lung cancer.

The statistical analysis revealed no significant difference in expression between normal and cancer tissues $(\mathrm{P}=0.995)$.

Histological classification of the lung cancer tissues was performed in order to identify differential expression of KLF4 between NSCLC and SCLC and between each tumor type and normal tissue. The statistical analysis revealed a significant difference in KLF4 expression between NSCLC and SCLC samples $(\mathrm{P}<0.0001)$. Of the 31 NSCLC cases, $19(61 \%)$ were negative for KLF4 and 12 (39\%) were positive. All 16 SCLC cases were positive for KLF4 [11 cases exhibited intense staining (3+) and 5 cases moderate staining (2+)]. The overall score of KLF4 expression was $0.7 \pm 0.3$ and $2.68 \pm 0.46$ in NSCLC and SCLC, respectively. A significant difference was also observed between normal tissues and each of the cancer tissue types ( $\mathrm{P}=0.00003$ for SCLC and $\mathrm{P}=0.02$ for NSCLC).

In NSCLC, a significant difference in KLF4 expression was observed between stage I and stages II, III and IV

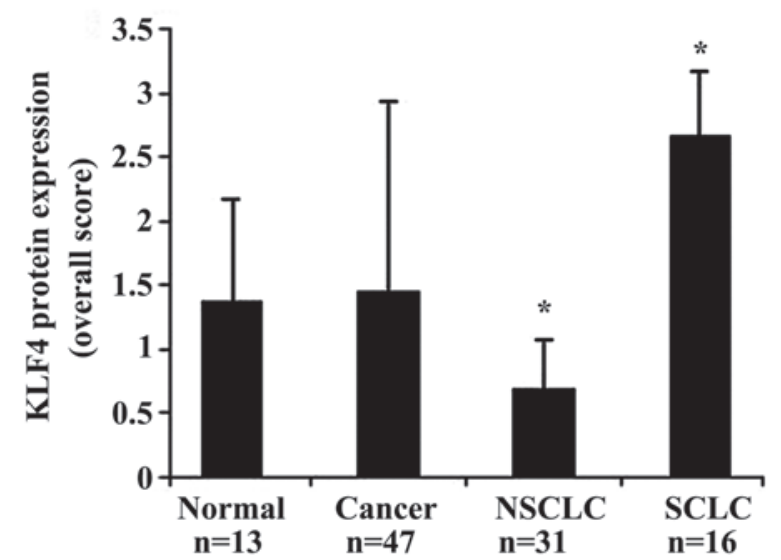

Figure 1. Krüppel-like factor 4 (KLF4) expression in normal and cancer tissues. The KLF4 protein expression levels were significantly decreased in non-small-cell lung cancer (NSCLC) tissues and significantly increased in small-cell lung cancer (SCLC) tissues compared with normal tissues $\left({ }^{*} \mathrm{P}<0.05\right)$.

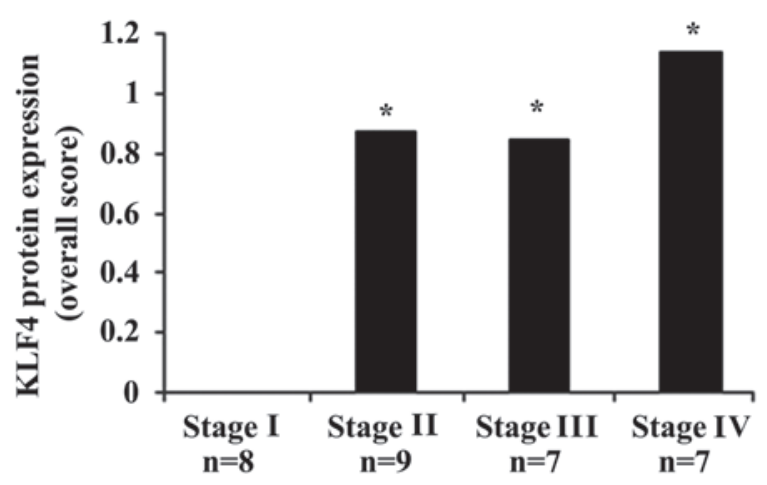

Figure 2. Krüppel-like factor 4 (KLF4) expression in different stages of non-small-cell lung cancer. KLF4 expression was significantly increased in tumor stages II, III and IV compared with stage I ( $\mathrm{P}<0.05)$.

$(\mathrm{P}<0.05)$ (Fig. 2). KLF4 expression was significantly increased in tumor stages II, III and IV, whereas all stage I cases $(n=8)$ were negative for KLF4 expression. The profile of KLF4 expression in each tumor stage is shown in Table III and examples of the immunohistochemical staining for KLF4 are shown in Fig. 3.

Factors associated with KLF4 protein level. In order to determine any correlation of KLF4 expression with age and gender, a statistical analysis was performed; KLF4 expression was not found to be significantly associated with age or gender.

\section{Discussion}

The identification of proteins or transcription factors with altered expression as a manifestation of human lung carcinogenesis is important in the discovery of biomarkers for early detection of lung cancer. Only a limited number of studies have analyzed the expression and role of KLF4 in lung cancer $(16,23-26)$. In the present study, we investigated the KLF4 protein expression in a series of human lung tumors and normal tissues. As a result, differential expression was observed between healthy tissue and each of the two major lung cancer 
Table III. Profile of Krüppel-like factor 4 protein expression in each tumor stage (cases per intensity of expression).

\begin{tabular}{lcccc}
\hline & \multicolumn{4}{c}{ Expression, $\mathrm{n}(\%)$} \\
\cline { 2 - 5 } Stage & 0 (no staining) & 1+ (mild staining) & 2+ (moderate staining) & 3+ (intense staining) \\
\hline I $(\mathrm{n}=8)$ & $8(100.0)$ & & & \\
II $(\mathrm{n}=9)$ & $5(55.0)$ & $2(28.0)$ & $4(45.0)$ & \\
III $(\mathrm{n}=7)$ & $3(44.0)$ & $1(140)$ & $2(28.0)$ & $1(14.0)$ \\
IV $(\mathrm{n}=7)$ & $3(420)$ & & $2(28.0)$ & \\
\hline
\end{tabular}

\section{$\mathbf{A}$}

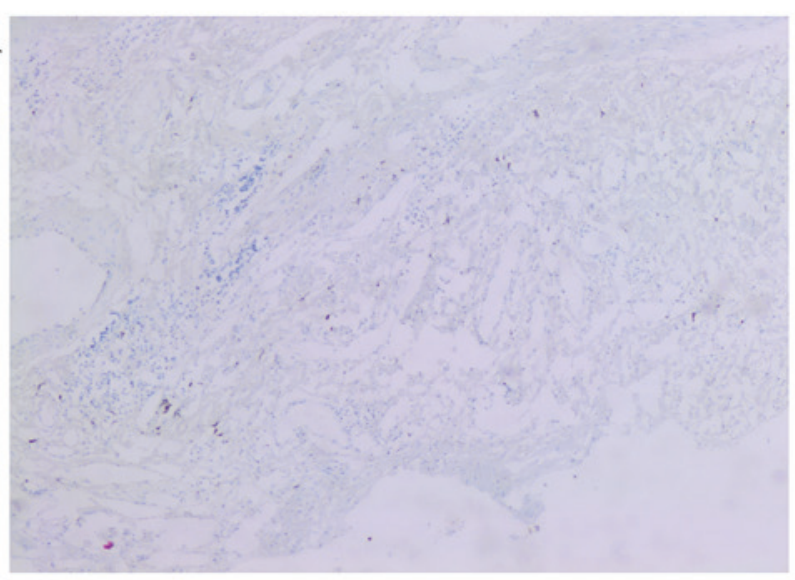

C

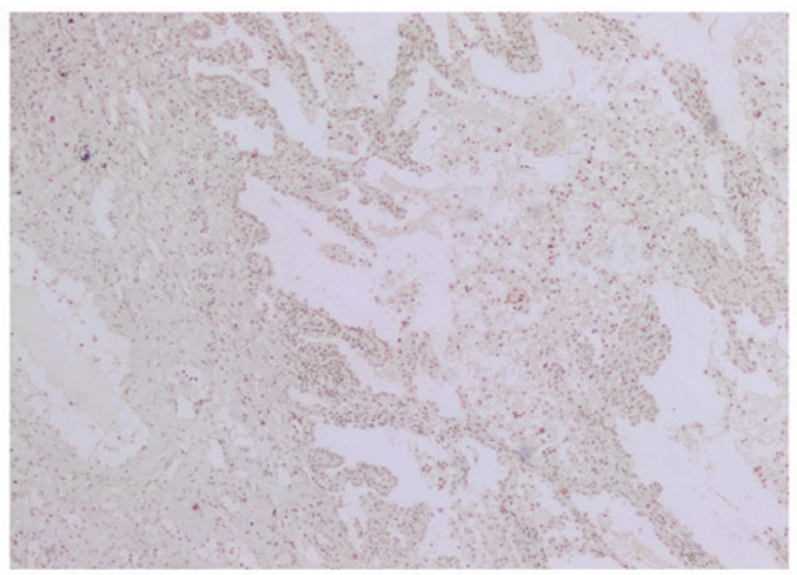

B

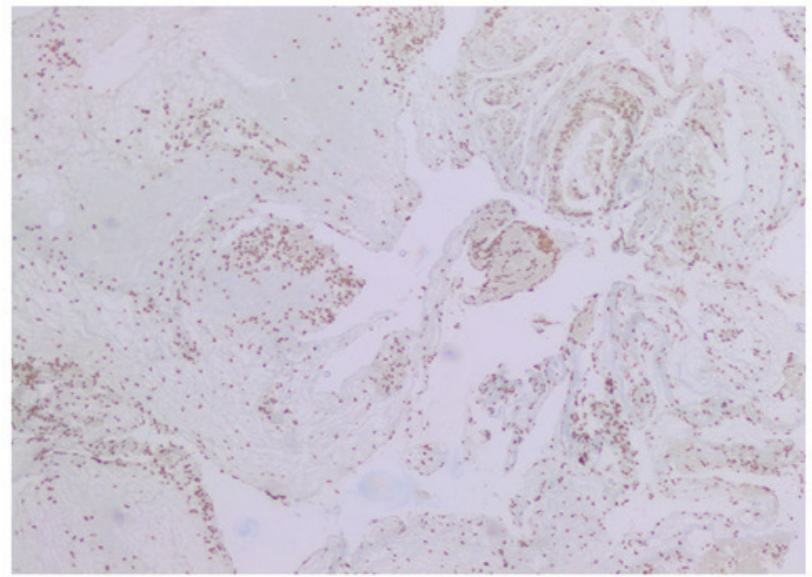

D

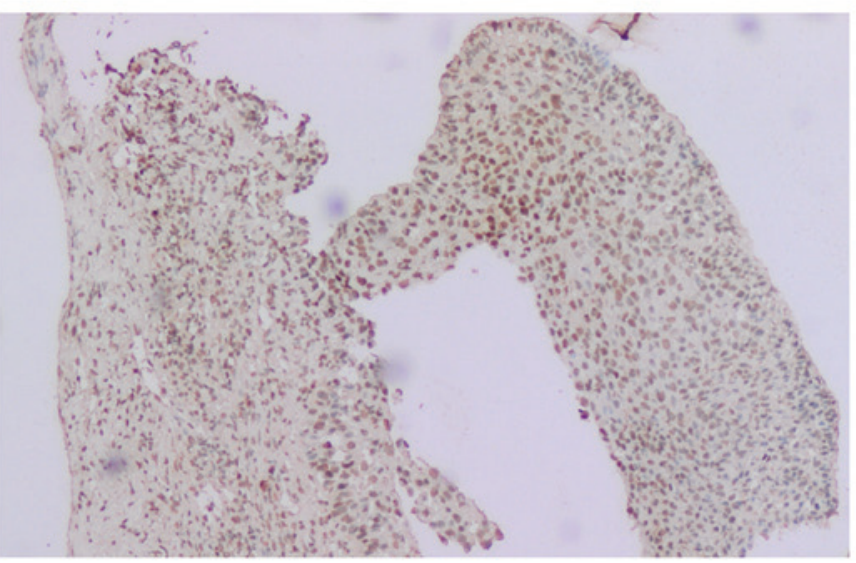

Figure 3. Analysis of Krüppel-like factor 4 expression by immunohistochemistry. Tissue sections with (A) 0 , no staining, (B) $1+$, mild staining, (C) $2+$, moderate staining and (D) $3+$, intense staining.

types (normal vs. NSCLC and normal vs. SCLC) and also between NSCLC and SCLC. The protein expression level of KLF4 was significantly decreased in NSCLC compared with that in normal tissue, while significant overexpression was detected in SCLC, which represents the fast-growing nature of this type of lung cancer that is considered highly lethal. These findings suggest that KLF4 may play a role in the carcinogenic process. Stage II, III and IV lung adenocarcinomas exhibited significantly higher rates of KLF4 expression compared with stage I disease, where the expression of KLF4 was absent. The absence of KLF4 expression may be explained by potential gene silencing due to hypermethylation. However, the mechanisms underlying this silencing require elucidation by future studies. The increase in KLF4 expression in stage II, III and IV disease may be associated with decreased tumor differentiation and increased aggressiveness.

Our findings provide preliminary data regarding the expression and the potential role of KLF4 in the proliferation of lung tumors depending on the cell type and context. Moreover, to the best of our knowledge, this is the first collected data showing a significant difference in KLF4 protein expression between the two major lung cancer types. However, due to the lack of patient survival data, we were unable to investigate any correlation between immunohistochemical findings and patient survival.

Our results were consistent with those reported by Naranjo Gómez et al showing high expression of KLF4 in neuroendocrine lung carcinomas, where KLF4 was 
positive in 23 of 35 large-cell neuroendocrine carcinomas, 10 of 10 tumorlets, 15 of 47 typical carcinoids and 18 of 18 SCLCs (25). Our results are also in agreement with those of Zhang et al, who demonstrated a reduction of KLF4 protein expression in NSCLC tumor specimens, compared with the expression in control tissues (26). However, in their study, Zhang et al did not evaluate the protein expression level of KLF4 in a SCLC tissue sample; therefore, a comparison of the KLF4 profile between the two major cancer types was lacking. The expression of KLF4 appears to exert a dual effect on lung cancer, depending on the cell context and gene network. Our actual in vitro study aims to determine the underlying mechanisms and the potential factors that regulate the gene or the protein expression of KLF4 in the two major histological groups of lung cancer. DNA mutations, molecular alterations, hypermethylation or microRNA expression may be associated with altered KLF4 expression in lung cancer types.

Our observations that KLF4 was increased in SCLC were not consistent with those of $\mathrm{Hu}$ et al, since our observations indicate that KLF4 may function as a tumor-promoting gene in lung cancer. The abovementioned studies have demonstrated that the expression of KLF4 is downregulated in a number of primary lung cancers and the ectopic expression of KLF4 suppresses lung cancer cell proliferation and tumor growth in vivo (16). This discrepancy may be due to the marginally larger tumor sample collection in our study, potentially contributing to more relevant results, and to the different method used to evaluate KLF4 expression. We examined the level of KLF4 protein expression by immunohistochemistry, whereas the level of KLF4 protein was measured by western blot analysis in the other study (16). However, the downregulation of KLF4 in NSCLC may be associated with promoter hypermethylation, a loss of heterozygosity of the KLF4 locus, or to point mutations in the coding region. How KLF4 is differentially expressed in lung cancers remains unclear. We hypothesized that epigenetic control and the gene network may play a role in the variable KLF4 expression levels in lung cancers and, therefore, requires further investigation.

KLF4 expression and its role in the two major types of lung cancer have not been extensively investigated to date. Furthermore, the molecular mechanisms underlying the tumor-suppressive or oncogenic function of KLF4 in lung cancer remain to be determined. It is important that the regulation of KLF4 expression in normal and tumor tissues is elucidated in future studies.

Our data suggest that KLF4 protein expression level in normal as well as tumor tissues may be a potential biomarker in patients with lung cancer. Our findings may be useful for determining prognostic factors associated with lung cancer and for supporting their possible use in lung cancer case stratification. In addition, SCLCs present with a more aggressive clinical course. Investigating how the microenvironment and cell context affect KLF4 expression and, thus, tumorigenesis, tumor progression and prognosis, is a major goal in future studies.

\section{Acknowledgements}

The present study was supported by a grant from the Lebanese University.

\section{References}

1. Molina JR, Yang P, Cassivi SD, Schild SE and Adjei AA: Non-small-cell lung cancer: Epidemiology, risk factors, treatment and survivorship. Mayo Clin Proc 83: 584-594, 2008.

2. Travis WD, Travis LB and Devesa SS: Lung cancer. Cancer 75 (1 Suppl): S191-S202, 1995

3. Preiss A, Rosenberg UB, Kienlin A, Seifert E and Jäckle H: Molecular genetics of Krüppel, a gene required for segmentation of the Drosophila embryo. Nature 313: 27-32, 1985.

4. Dang DT, Pevsner J and Yang VW: The biology of the mammalian Krüppel-like family of transcription factors. Int J Biochem Cell Biol 32: 1103-1121, 2000.

5. Black AR, Black JD and Azizkhan-Clifford J: Sp1 and Krüppel-like factor family of transcription factors in cell growth regulation and cancer. J Cell Physiol 188: 143-160, 2001.

6. Kaczynski J, Cook T and Urrutia R: Sp1- and Krüppel-like transcription factors. Genome Biol 4: 206, 2003.

7. Garrett-Sinha LA, Eberspaecher H, Seldin MF and de Crombrugghe B: A gene for a novel zinc-finger protein expressed in differentiated epithelial cells and transiently in certain mesenchymal cells. J Biol Chem 271: 31384-31390, 1996.

8. Shields JM, Christy RJ and Yang VW: Identification and characterization of a gene encoding a gut-enriched Krüppel-like factor expressed during growth arrest. J Biol Chem 271: 20009-20017, 1996.

9. Zhao W, Hisamuddin IM, Nandan MO, Babbin BA, Lamb NE and Yang VW: Identification of Krüppel-like factor 4 as a potential tumor suppressor gene in colorectal cancer. Oncogene 23: 395-402, 2004

10. Wei D, Gong W, Kanai M, Schlunk C, Wang L, Yao JC, Wu TT, Huang S and Xie K: Drastic down-regulation of Krüppel-like factor 4 expression is critical in human gastric cancer development and progression. Cancer Res 65: 2746-2754, 2005.

11. Wei D, Kanai M, Huang S and Xie K: Emerging role of KLF4 in human gastrointestinal cancer. Carcinogenesis 27: 23-31, 2006.

12. Nakahara Y, Northcott PA, Li M, Kongkham PN, Smith C, Yan H, Croul S, Ra YS, Eberhart C, Huang A, et al: Genetic and epigenetic inactivation of Kruppel-like factor 4 in medulloblastoma. Neoplasia 12: 20-27, 2010.

13. Yasunaga J, Taniguchi Y, Nosaka K, Yoshida M, Satou Y, Sakai T, Mitsuya H and Matsuoka M: Identification of aberrantly methylated genes in association with adult T-cell leukemia. Cancer Res 64: 6002-6009, 2004.

14. Foster KW, Ren S, Louro ID, Lobo-Ruppert SM, McKie-Bell P, Grizzle W, Hayes MR, Broker TR, Chow LT and Ruppert JM: Oncogene expression cloning by retroviral transduction of adenovirus E1A-immortalized rat kidney RK3E cells: Transformation of a host with epithelial features by c-MYC and the zinc finger protein GKLF. Cell Growth Differ 10: 423-434, 1999.

15. Wang N, Liu ZH, Ding F, Wang XQ, Zhou CN and Wu M: Down-regulation of gut-enriched Kruppel-like factor expression in esophageal cancer. World J Gastroenterol 8: 966-970, 2002.

16. Hu W, Hofstetter WL, Li H, Zhou Y, He Y, Pataer A, Wang L, Xie K, Swisher SG and Fang B: Putative tumor-suppressive function of Kruppel-like factor 4 in primary lung carcinoma. Clin Cancer Res 15: 5688-5695, 2009.

17. Ohnishi S, Ohnami S, Laub F, Aoki K, Suzuki K, Kanai Y, Haga K, Asaka M, Ramirez F and Yoshida T: Downregulation and growth inhibitory effect of epithelial-type Krüppel-like transcription factor KLF4, but not KLF5, in bladder cancer. Biochem Biophys Res Commun 308: 251-256, 2003.

18. Foster KW, Frost AR, McKie-Bell P, Lin CY, Engler JA, Grizzle WE and Ruppert JM: Increase of GKLF messenger RNA and protein expression during progression of breast cancer. Cancer Res 60: 6488-6495, 2000.

19. Pandya AY, Talley LI, Frost AR, Fitzgerald TJ, Trivedi V, Chakravarthy M, Chhieng DC, Grizzle WE, Engler JA, Krontiras $\mathrm{H}$, et al: Nuclear localization of KLF4 is associated with an aggressive phenotype in early-stage breast cancer. Clin Cancer Res 10: 2709-2719, 2004.

20. Chen YJ, Wu CY, Chang CC, Ma CJ, Li MC and Chen CM: Nuclear Krüppel-like factor 4 expression is associated with human skin squamous cell carcinoma progression and metastasis. Cancer Biol Ther 7: 777-782, 2008.

21. Foster KW, Liu Z, Nail CD, Li X, Fitzgerald TJ, Bailey SK, Frost AR, Louro ID, Townes TM, Paterson AJ, et al: Induction of KLF4 in basal keratinocytes blocks the proliferation-differentiation switch and initiates squamous epithelial dysplasia. Oncogene 24: 1491-1500, 2005. 
22. Huang CC, Liu Z, Li X, Bailey SK, Nail CD, Foster KW, Frost AR, Ruppert JM and Lobo-Ruppert SM: KLF4 and PCNA identify stages of tumor initiation in a conditional model of cutaneous squamous epithelial neoplasia. Cancer Biol Ther 4: $1401-1408,2005$

23. Yu T, Chen X, Zhang W, Liu J, Avdiushko R, Napier DL, Liu AX, Neltner JM, Wang C, Cohen D and Liu C: KLF4 regulates adult lung tumor-initiating cells and represses K-Ras-mediated lung cancer. Cell Death Differ 23: 207-215, 2016.

24. Zhou Y, Hofstetter WL, He Y, Hu W, Pataer A, Wang L, Wang J, Zhou Y, Yu L, Fang B and Swisher SG: KLF4 inhibition of lung cancer cell invasion by suppression of SPARC expression. Cancer Biol Ther 9: 507-513, 2010 .
25. Naranjo Gómez JM, Bernal JF, Arranz PG, Fernández SL and Roman JJ: Alterations in the expression of p53, KLF4 and p21 in neuroendocrine lung tumors. Arch Pathol Lab Med 138: 936-942, 2014.

26. Zhang Z, Wang Z, Liu X, Shi M, Chen G, Zhang B, Li Z and Song L: Correlation of KLF4 and SPARC expression with the clinical characteristics of non-small cell lung cancer. Chin J Lung Cancer: 720-724, 2012 (In Chinese). 